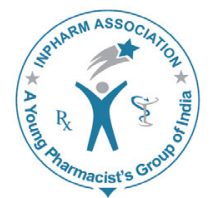

\title{
Oral Rehabilitation of a Child with Goldenhar syndrome
}

\author{
Sai Sankar Avula Jogendra, Sujatha Bandi, Satya Gopal Akkla* and Mahesh Chinta \\ Department of Pedodontics and Preventive Dentistry, Sibar Institute of Dental Sciences, Guntur, A.P. INDIA.
}

\begin{abstract}
Goldenhar syndrome (GS) is a rare abnormality affecting the craniofacial region having extra cranial manifestations as well. The first observation of the anomaly was reported by Arlt F in 1881. Dr Maurice Goldenhar in 1952 gave the name Goldenhar syndrome. The etiology of this disease still remains unclear and occurs as sporadic. However, some researchers have suggested multifactorial inheritance caused by the interaction of many genes, possibly in combination with environmental factors. The incidence has been reported to range from 1 in 3,500 to 1 in 5,600 live births; however, in children with congenital deafness, it is much higher at 1 in 1000. Patients with this syndrome may exhibit a wide range of anomalies that includes eye anomalies (micropthalmos, coloboma, epibulbar dermoid and lipodermoid malformations); ear anomalies (microtia, atresia, ear appendices and fistulas); vertebral and congenital heart anomalies; cleft lip/cleft palate, facial asymmetry, developmental dental disturbances and mental retardation. VATER (Vertebral anomalies, Anal atresia, Tracheoesophageal fistula, Esophageal atresia, Renal anomalies and Radial Dysplasia) or CHARGE (Coloboma, Heart defects, Atresia chooanae. This case report presents the dental management of a 7 year old boy with characteristic combination of external ear anomalies and ipsilateral facial underdevelopment the hallmark of this syndrome.
\end{abstract}

Key words: Corneal opacities, Goldenhar syndrome, Mandibular hypoplasia, Oral Rehabilitation, Periauricular tags.

\section{INTRODUCTION}

Franceschetti-Goldenhar syndrome or Goldenhar syndrome, also known as facio-auriculo-vertebral spectrum (FAV), first and second branchial arch syndrome, or oculoauriculovertebral (OAV) spectrum is a rare congenital malformation which encompasses various morphological and functional abnormalities. ${ }^{1,2}$ This syndrome was first reported by German physician Carl Ferdinand Von Arlt in 1845, however, when Maurice Goldenhar described its various characteristic features in 1952, the credit of discovery went to him.,

\begin{tabular}{|l|l|}
\hline \multicolumn{2}{|c|}{ Access this article online } \\
\hline \multirow{2}{*}{ Journal Sponsor } & \multirow{2}{*}{ Website: } \\
www.jphoungpharm.org \\
& \\
\cline { 2 - 2 } & DOI: \\
& $10.5530 /$ jyp.2015.3.21 \\
& \\
\hline
\end{tabular}

Goldenhar syndrome (GS) is sporadic and its etiology is not fully understood. However, positive family history has been reported suggesting auto so mal dominant or recessive inheritance. Some researchers have suggested multifactorial inheritance caused by the interaction of many genes, possibly in combination with environmental factors. ${ }^{2,3,5}$ The incidence has been reported to range from 1 in 3,500 to 1 in 5,600 live births; however, in children with congenital deafness, it is much higher at 1 in 1000. There is a male predominance of the syndrome at a ratio of $3: 2 .^{6}$

Goldenhar syndrome (GS) is typically associated with the anomalous development of structures that originate from the first and second branchial arches. The affected individual's face may appear smaller on one side than the other due to craniofacial malformations. Patients with this syndrome may exhibit a wide range of anomalies that includes eye anomalies (micropthalmos, coloboma,

\footnotetext{
*Address for correspondence:

Dr. Satya Gopal Akkla M. D. S, Assistant Professor, Department of Pedodontics and Preventive Dentistry, Sibar Institute of Dental

Sciences, Guntur, A.P. India. Email: satyagopal.akkala@gmail.com
} 

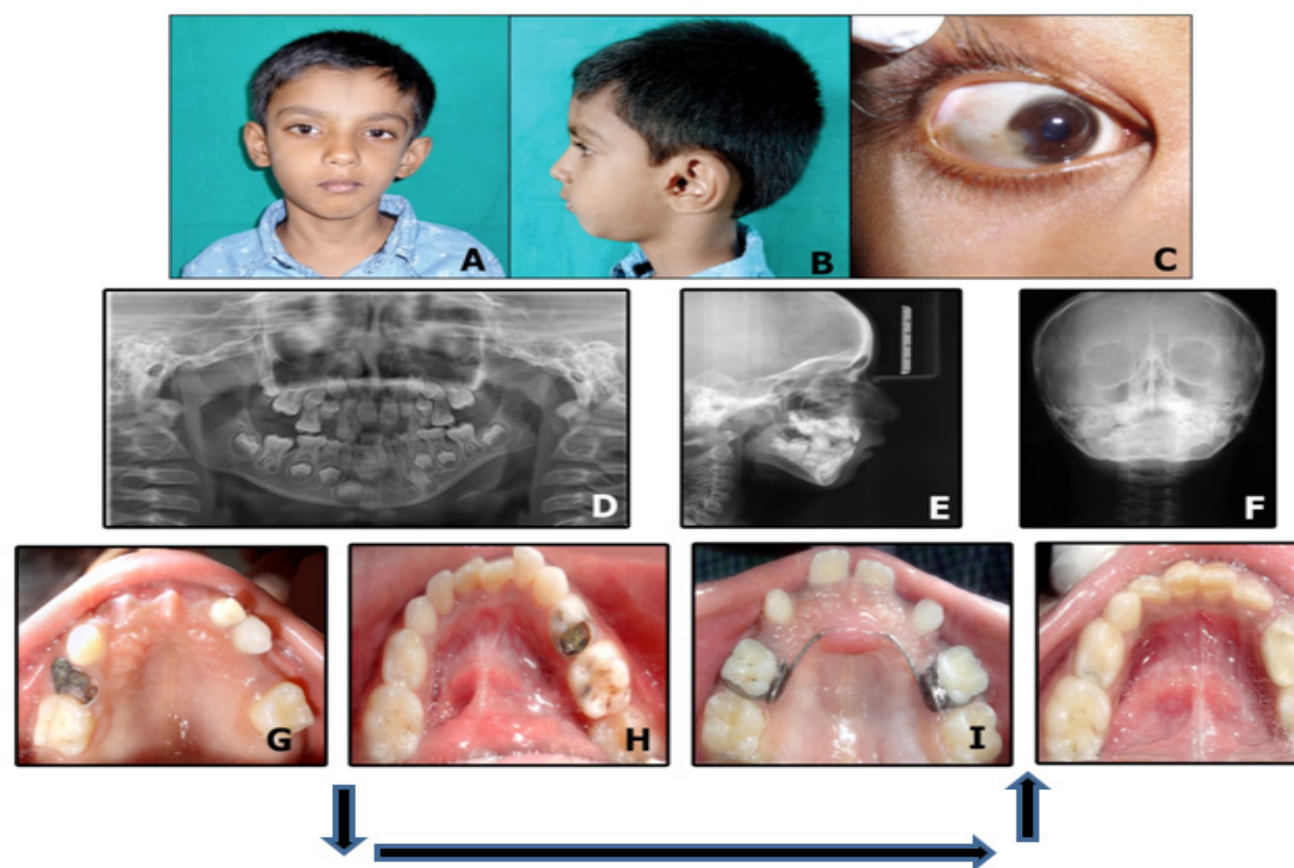

Graphical Abstract

Sequential Images of the Classical features, Radiographic findings and Oral rehabilitation of a child with Goldenhar Syndrome.

epibulbar dermoid and lipodermoid malformations); ear anomalies (microtia, atresia, ear appendices and fistulas); vertebral and congenital heart anomalies; cleft lip/cleft palate, facial asymmetry, developmental dental disturbances and mental retardation. ${ }^{7,8}$

This present case report illustrates the oral rehabilitation of a child with Goldenhar syndrome (GS) with characteristic combination of external ear anomalies and ipsilateral facial underdevelopment which are the hallmark of this syndrome.

\section{CASE REPORT}

A 7 year old boy reported to the department of Pediatric dentistry with a complaint of decayed teeth in upper and lower left back tooth region. The patient was born to nonconsanguineous parents and his elder sibling was apparently normal. History revealed that his birth was uneventful. Medical history revealed that the child underwent surgery for reconstruction of both auricles at the age of 2 weeks and corneal transplant surgery with regard to the left eye anomalies at the age of 4 years. However, there was no delay in developmental milestones as reported by the parents.

On extra oral examination, the patient presented with facial asymmetry, with deviation of the face to the left; mandibular hypoplasia and loss of malar prominence on the affected side. There was marked deviation of the nasal septum towards the left side, the left orbit was smaller in size with corneal opacities in the right eye. Hearing was normal with a soft tissue deformity near left ear. Facial profile was convex and submandibular lymphadenopathy is absent. There are no signs of mental retardation or neurological problems (Figure 1A-1J).

Intraoral examination showed a mixed dentition stage with mesial step molar relation and crowding of mandibular anterior teeth. On opening there is a deviation to the left and protrusive movement lead deviation of the tongue to the left. Even though the anatomy of the tongue is normal there was papillar atrophy of the tongue in the anterior third. The level of attachment of the maxillary and mandibular fraenula appeared normal. The child had chronic generalized gingivitis which is associated with poor oral hygiene and carious lesions of varying severity in relation to 5264 and 74 region with missing 54 .

Panoramic radiograph revealed hypoplasia of the condyle, ramus and body of the mandible with accentuation of the antegonial notch on the left side. Lateral cephalogram showed a high mandibular angle with a clockwise rotation indicating increased vertical growth. Water's view revealed deformity of the left orbit and a deviated nasal septum toward the right. No vertebral anomalies were diagnosed by the radiologist in the anterio-posterior and lateral views of thoracic lumbar spine and cervical spine.

The child was referred to an ophthalmologist, a cardiologist 

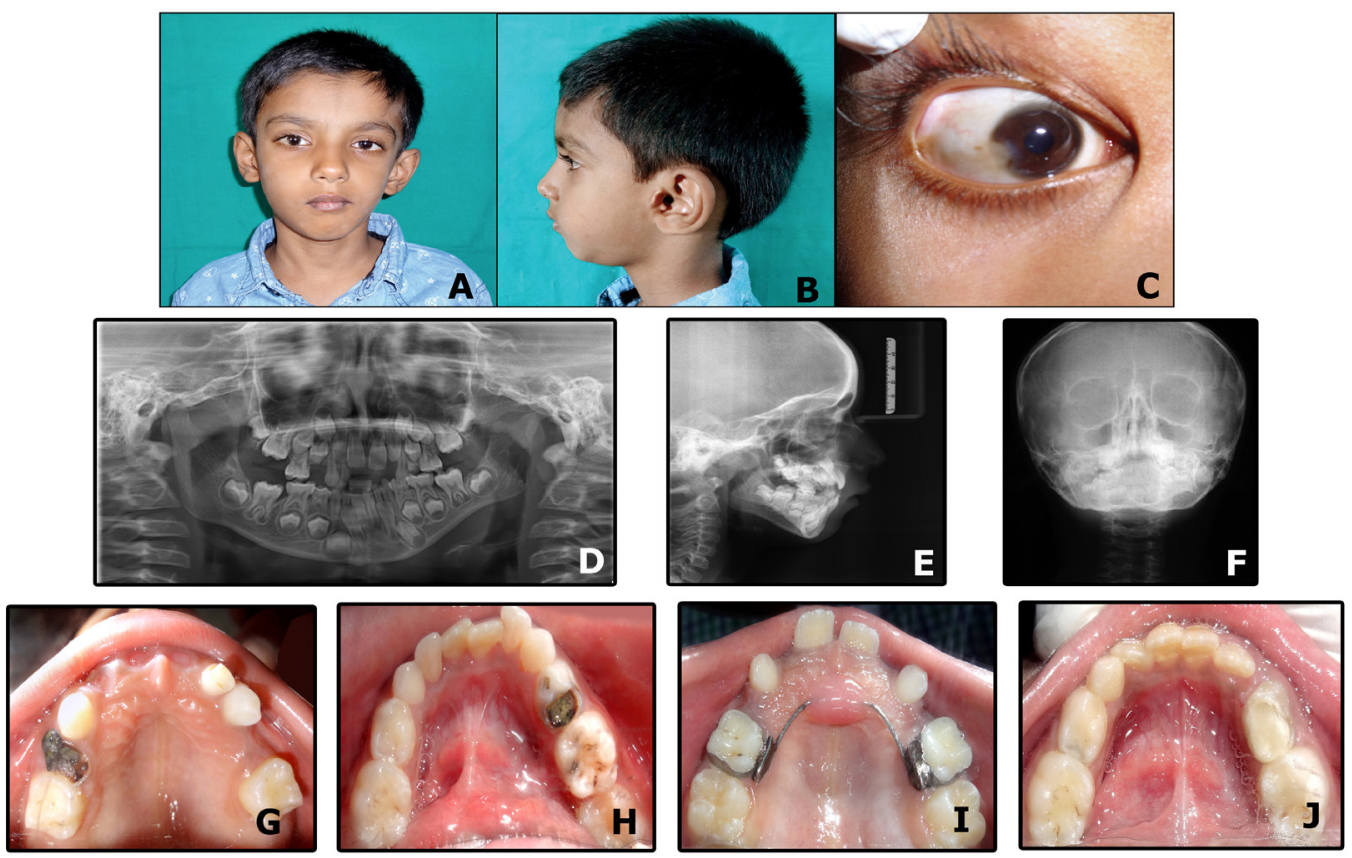

Figure 1A: Extra oral photograph showing facial asymmetry. Figure 1B: Extra oral photograph showing preauricular ear tag. Figure 1C: Extra oral photograph showing corneal opacity. Figure 1D: OPG showing hypoplasia of the condyle, ramus, and body of the mandible with accentuation of antegonial notch on the left side. Figure 1E: Lateral cephalogram demonstrating a high mandibular angle with a clockwise rotation. Figure 1F: Water's view $X$ ray revealing deformity of the right orbit and deviated nasal septum toward the right. Figure 1G, 1H 1I, 1J: Pre and post operative photographs.

and an ENT surgeon for further investigation. On ophthalmic examination, the patient was diagnosed with vascularised corneal opacity with loss of corneal sensation in the right eye. Nevertheless his vision was within normal range. Routine cardiac examination and an echocardiogram showed no cardiac abnormality. Otic examination showed preauricular tags of cartilaginous masses on left side; however, there was no deformity in the ear canal. Based on the overall clinical, radiographic and investigative findings a confirmatory diagnosis of Goldenhar syndrome was made.

The dental treatment plan was based on the patient's caries risk and consisted of restoration of carious teeth and extraction of unrestorable primary tooth followed by space management. As there were no systemic abnormalities, antibiotic prophylaxis was not recommended. Oral hygiene was improved through professional oral prophylaxis, home care instructions and restoration of carious teeth. Pulpectomy was carried out in relation to 74 followed by permanent restoration with glass ionomer cement and stainless steel crown was not placed as the root length was minimal. 64 was extracted and Nance palatal arch space maintainer was delivered as there was missing 54 and 52 was restored with composite restoration. As the child is in growth phase the, extensive orthodontic intervention including surgical correction of jaw deformity was suggested only after growth cessation.

\section{DISCUSSION}

In 1952, Goldenhar described a patient with a triad of accessory tragic, mandibular hypoplasia and ocular dermoids which he called as the Goldenhar syndrome. In 1963, Gorlin named this syndrome as oculo auriculovertebral as it consists classically the triad of mal-development of the first and second branchial arches, occular dermoids and vertebral anomalies (usually unilateral). ${ }^{4}$ Other conditions like VATER (Vertebral anomalies, Anal atresia, Tracheoesophageal fistula, Esophageal atresia, Renal anomalies and Radial Dysplasia) or CHARGE (Coloboma, Heart defects, Atresia chooanae, Retardation of growth and development, Genital and urinary abnormalities and Ear abnormalities and/or hearing loss) also resemble GS in their clinical manifestations, connection among these anomalies could be a common pathogenetic mechanism namely, disturbed neural crest development.'

The study of this condition is still controversial because the symptoms and the physical features may vary greatly in range and severity from case to case. The characteristic combination of external ear anomalies and ipsilateral facial underdevelopment is the hallmark of this syndrome ${ }^{10}$ as noticed in our patient. In most cases, such malformations affect one side of the body; however, in $10-33 \%$ of affected individuals, both sides of the body are involved 
with one side typically more affected than the other. In the present case left side was affected, however in previous reports the right side is more severely affected than the left. ${ }^{7}$

Exact etiology remains unclear but genetic causes and vascular changes like fetal hemorrhage in the region of first and second arches at the time when the blood supply switches from stapedial artery to external carotid artery were suggested. ${ }^{11}$ Disturbance in neural crest development, maternal diabetes, rubella and influenza have also been implicated. ${ }^{3,9}$ In the present case also the etiology seemed unclear.

In the present case, marked malar prominence and mandibular hypoplasia with mal-alignment of the teeth and preauricular tags are among the various craniofacial anomalies that were reported. No eye anomalies were present except corneal opacity; this might be due to the corneal transplant surgery done previously. Although the vertebral anomalies may include hypoplasia, fusion or absence of certain vertebra, no vertebral anomalies were detected in our patient. ${ }^{12}$ Despite the reported frequency of cardiovascular alterations ranging from 5 to $58 \%,{ }^{13}$ no cardiac abnormality was found in this patient.

Due to the complexity and varying severity in expression, management of GS requires a multidisciplinary approach. As the child grows, the effects of GS may become more pronounced since the affected areas will show a significant delay in development. This can result in cosmetic concerns, breathing difficulties and malocclusion, putting them at increased risk of dental diseases. Hence periodic dental visits with emphasis on preventive strategies like proper brushing technique and fluoride application are important considerations in these individuals.

Hypoplasia of the jaw bone in this patient can be corrected by distraction osteogenesis and reconstruction with bone grafts. Structural anomalies of eyes and ears can be corrected by reconstructive surgery as done in this present case at an early age which minimizes the esthetic concern. These individuals usually have a normal lifespan and require a long-term periodic follow-up to monitor the child's growth and development.

\section{CONCLUSION}

The study of this condition is still controversial because of its complexity and broad clinical aspects. This case report illustrates various etiological factors, classical features, diagnosis, dental considerations and management of patients of pediatric age group.

\section{ACKNOWLEDGEMENTS}

We Acknowledgement patient and the parent for their cooperation during treatment and follow-up visits. We sincerely thank Dr M G Manoj Kumar and Dr D Mahesh Kumar, for helping out in premaring the manuscript.

\section{Highlights of Paper}

- Illustrates various etiological factors, classical features and diagnosis of Goldenhar Syndrome.

- Early management of dental problems in children to prevent the complications at a later age.

- Emphasis on preventive strategies for dental diseases.

\section{Author Profile}

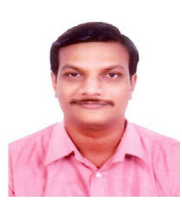

- Dr Avula Jogendra Sai Sankar M.D.S: presently working as Professor and Head in the Department of PEDODONTICS \& PREVENTIVE DENTISTRY, at Sibar Institute of Dental Sciences, Guntur, Andhra Pradesh. He has 28 nos. of journal paper published and is a Reviewer and Editorial Board member, Turkiye Klinikleri Journal of Dental Sciences (Turkey) and Peer Reviewer for - Journal of Dentistry and Oral hygiene (JDOH); International Journal of Clinical Pediatric Dentistry (IJCPD); Journal of Orofacial Sciences (JOFS); Journal of Clinical and Diagnostic Research (JCDR); Journal of Oral Research and Review (JORR).

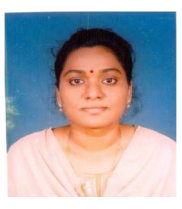

- Dr. B.SUJATHAM.D.S: presently working as Professor in the Department of PEDODONTICS \& PREVENTIVE DENTISTRY, at Sibar Institute of Dental Sciences, Guntur, Andhra Pradesh. She has 10 nos. of journal paper published and is a Regular participant in all CDE programs, State and National level Conferences, Symposiums, Specialty conferences and Workshops. 


\section{REFERENCES}

1. Subramaniam P, Girish Babu KL, Jayasurya S, Prahalad D. Dental management of a child with Goldenhar syndrome. Eur J Gen Dent. 2014; 3(2): 158-62.

2. Tuna EB, Orino D, Ogawa K, Yildirium M, Seymen F, Gencay K. Craniofacial and dental characteristics of Goldenhar syndrome: A report of two cases. J Oral Sci. 2011; 53(1): 121-4.

3. Mahore A, Dange N, Nama S, Goel A. Facio-auriculo-vertebrocephalic spectrum of Goldenhar syndrome. Neurol India. 2010; 58(1): 141-4.

4. Kulkarni VV, Shah MD, Parikh AA. Goldenhar syndrome: A case report. J Postgrad Med. 1985; 31(3): 177-9.

5. Gorlin R, Jue K, Jacohsen U, Goldschmidt E. Oculoauriculovertebral dysplasia. J Pediatr. 1963; 63(5): 991-9.

6. Kokavec R. Goldenhar syndrome with various clinical manifestations. Cleft Palate Craniofac J. 2006; 43(5): 628-34.

7. Pierpont ME, Moller JH, Gorlin RJ, Edwards JE. Congenital cardiac, pulmonary and vascular malformations in oculoauriculovertebral dysplasia. Pediatr Cardiol 1982; 2(4): 297-302.
8. Bayraktar S, Bayraktar ST, Ataoglu E, Ayaz A, Elevli M. Goldenhar's syndrome associated with multiple congenital anomalies. J Trop Paediatr. 2005; 51(6): 377-9.

9. Kallen K, Robert E, Castilla EE, Mastroiacovo P, Källén B. Relation between oculo-auriculo-vertebral (OAV) dysplasia and three other nonrandom associations of malformations (VATER, CHARGE, and OEIS). Am J Med Genet A. 2004; 127(1): 26-34.

10. Grabb WC. The first and second brachial arch syndromes. Plast Reconstr Surg. 1965; 36(5): 485-508.

11. Soltan HC, Holmes LB. Familial occurrence of malformations possibly attributable to vascular abnormalities. J Pediatr. 1986; 108(1): 112-4.

12. Trivedi HL, Murade S, Harne S, Aarbhave V. Incomplete presentation of Goldenhar syndrome. Bombay Hosp J. 2007; 49: 519-21.

13. Nakajima H, Goto G Tanaka N, Ashiya H, Ibukiyama C. Goldenhar Syndrome associated with various cardiovascular malformations. Jpn Circ J. 1998; 62(8): 617-20. 